\title{
Hepatocyte growth factor upregulates nexilin gene expression in cardiomyocytes via JNK pathway
}

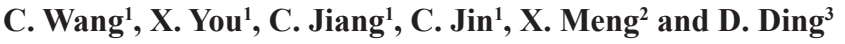 \\ ${ }^{1}$ Department of Laboratory Medicine, Affiliated Hospital of Yanbian University, \\ Yanji, China \\ ${ }^{2}$ Core Laboratory, Cardiovascular Institute and Fu Wai Hospital, \\ Chinese Academy of Medical Sciences and Peking Union Medical College, \\ Beijing, China \\ ${ }^{3}$ Department of Cardiology, Affiliated Hospital of Yanbian University, \\ Yanji, China \\ Corresponding author: X. You \\ E-mail: xinyou118@126.com
}

Genet. Mol. Res. 13 (3): 4976-4982 (2014)

Received May 28, 2013

Accepted November 2, 2013

Published July 4, 2014

DOI http://dx.doi.org/10.4238/2014.July.4.12

\begin{abstract}
Hepatocyte growth factor (HGF) is a protective factor in myocardial injury, but its mechanisms of action have not yet been fully elucidated. Nexilin, which locates specifically to the Z-disc, is a novel Z-disc protein that enables the Z-discs to persistently withstand the extreme mechanical forces generated during muscle contraction. Therefore, we investigated the role of HGF in modulating nexilin expression in hypoxia-reoxygenation (H/R)treated cardiomyocytes. We cultured neonatal cardiomyocytes and treated them with HGF. The mRNA and protein levels of nexilin were determined by RT-PCR and Western blotting. H/R treatment decreased nexilin mRNA expression and nexilin protein levels in cardiomyocytes. Furthermore, treatment with HGF upregulated nexilin expression and the JNK inhibitor SP600125 partly inhibited
\end{abstract}


HGF-induced nexilin upregulation. In conclusion, our results suggest that ischemia-reperfusion injury may downregulate nexilin expression in cardiomyocytes, and HGF may exert its protective role during myocardial ischemic injury through upregulation of nexilin expression in cardiomyocytes.

Key words: Hepatocyte growth factor; Nexilin; Cardiomyocytes; Hypoxia-reoxygenation

\section{INTRODUCTION}

Hepatocyte growth factor (HGF), which is originally identified and cloned as a mitogen for primary hepatocytes (Nakamura et al., 1989; Ozaki et al., 2002), can regulate cell growth, motility, and morphogenesis of various types of cells via c-Met/HGF receptor tyrosine kinase. HGF is also able to prevent fibrosis in the lung and liver models (Matsuda et al., 1997; Yaekashiwa et al., 1997). Interestingly, HGF can protect against myocardial injury through its angiogenetic and antifibrotic actions (Taniyama et al., 2002). HGF also protects myocardium from ischemia/reperfusion injury through its antiapoptotic effect on cardiomyocytes (Nakamura et al., 2000; Chen et al., 2007). Administration of HGF reduces infarct size and increases cardiac performance in a rat model of acute ischemia/reperfusion (Nakamura et al., 2000). Although these studies suggest a protective role of HGF in the myocardial injury, the exact mechanisms have not been clearly understood.

Nexilin, comprising 675 amino acids, was previously recognized as an F-actin-binding protein at cell-matrix adherens junctions (Ohtsuka et al., 1998). Recently, it has been shown that nexilin is highly expressed in the heart and skeletal muscle and locates specifically to the Z-disc and that the deficiency or mutations of nexilin lead to perturbed Z-disc stability and heart failure (Hassel et al., 2009). Therefore, nexilin is considered as a novel Z-disc protein that enables the Z-discs to persistently withstand the extreme mechanical forces generated during muscle contraction (Hassel et al., 2009). Moreover, 1 deletion and 2 missense mutations in NEXN have been identified in a large cohort of patients with dilated cardiomyopathy (Hassel et al., 2009). Wang et al. (2010) showed a correlation between nexilin mutation and hypertrophic cardiomyopathy (HTC).

In view of the unique role of nexilin in stabilizing and protecting Z-discs from mechanical trauma, the involvement of nexilin in the protective effect of HGF on cardiac function in ischemia/reperfusion injury remains unknown. Therefore, we aimed to observe the effect of HGF on nexilin expression in cardiomyocytes and to understand the potential mechanisms.

\section{MATERIAL AND METHODS}

\section{Reagents}

Dulbecco's modified Eagle's medium (DMEM) was purchased from Gibco (Life Technologies, Carlsbad, CA, USA). Enhanced chemiluminescent (ECL) substrate was purchased from ShineGene Bio-Technologies, Inc. (Shanghai, China). Polyvinylidene 
difluoride (PVDF) membrane was purchased from Millipore Corporation (Billerica, MA, USA). GAPDH antibodies were purchased from Serotec (Raleigh, NC, USA). Rabbit antimouse was purchased from Santa Cruz Biotechnology (Santa Cruz, CA, USA). M-MLV Reverse Transcriptase was purchased from Promega (Madison, WI, USA). TriPure reagent was purchased from Roche (Indianapolis, IN, USA).

\section{Cell culture and hypoxia-reoxygenation $(H / R)$ treatment}

Neonatal rat ventricular cardiomyocytes were isolated and cultured as described previously (Date et al., 2002). Briefly, the cells were seeded in rat type I collagen-coated dishes at a density of $2.0 \times 10^{5}$ cells $/ \mathrm{cm}^{2}$ and maintained in DMEM supplemented with $10 \%$ fetal bovine serum (FBS), at $37^{\circ} \mathrm{C}$ in a humidified incubator gassed with $95 \%$ air and $5 \% \mathrm{CO}_{2}$. After culture in normoxic DMEM for about 5-6 days, the medium was replaced with 1\% FBS-DMEM, and the cardiomyocytes were transferred to a hypoxic incubator that was continuously gassed with 5\% $\mathrm{CO}_{2}$ and $95 \% \mathrm{~N}_{2}\left(\mathrm{PO}_{2}\right.$ range maintained at $\left.<1 \%\right)$ for $24 \mathrm{~h}$, and then, the cells used for reoxygenation were set to a normal condition for the indicated times. Thus, hypoxic cells were grouped according to the time of reoxygenation. Corresponding control cells were planted with the same cell number as the hypoxic cells and maintained in a $95 \%$ air, $5 \% \mathrm{CO}_{2}$ incubator throughout the experiment.

\section{RT-PCR}

Total RNA was extracted from cardiomyocytes using TriPure reagent and reversetranscribed with M-MLV reverse transcriptase. The cDNA was subjected to PCR with 2 separate sets of oligonucleotide primers for nexilin and $\beta$-actin. PCR products were separated on $1 \%$ agarose gel and visualized with GelStar nucleic acid stain (Quantity One).

\section{Western blot analysis}

The cells were scraped off the plates and lysed in radioimmunoprecipitation assay (RIPA) buffer (50 mM Tris-HCl, pH 8.0, $150 \mathrm{mM} \mathrm{NaCl}, 1 \%$ Nonidet-P40, 0.5\% deoxycholic acid, and $0.1 \%$ sodium dodecyl sulfate). Cell lysates (50 $\mu \mathrm{g}$ protein) were electrophoresed on SDS-PAGE gel and transferred to a PVDF membrane. These membranes were incubated with antibodies against GAPDH or $\beta$-actin. After incubation, the corresponding secondary antibody signals were detected by the enhanced chemiluminescence reagents. The intensity of various protein bands was quantified by densitometry.

\section{Statistical analysis}

Data are reported as means \pm SD. Statistical significance was determined using SPSS 11.0 for Windows (SPSS Inc., Chicago, IL, USA). One-way ANOVA was performed for multiple comparisons followed by Fisher LSD post-hoc comparisons. Differences were deemed significant if $\mathrm{P}<0.05$. 


\section{RESULTS}

\section{H/R inhibited nexilin expression}

As shown in Figure 1A and B, the expression of nexilin was decreased after H/R. A time-dependent decrease in nexilin expression was also observed after $\mathrm{H} / \mathrm{R}$.
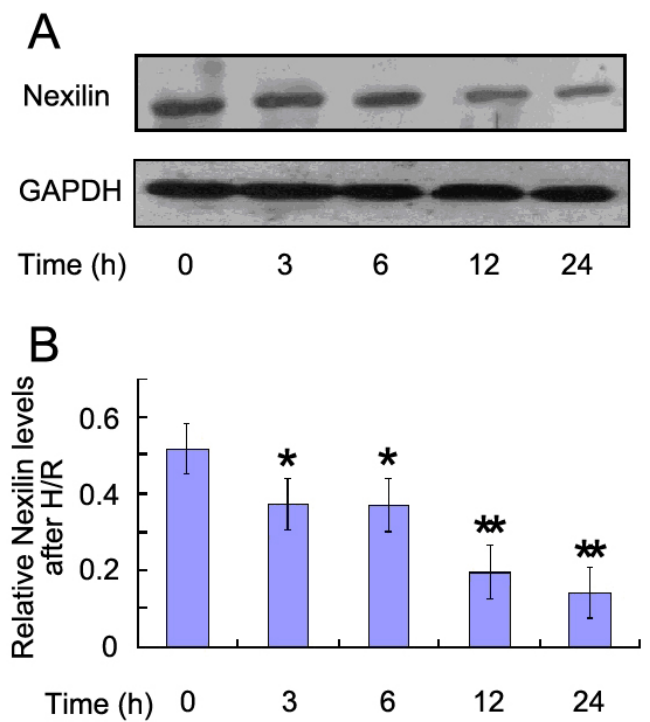

Figure 1. Nexilin was decreased in cardiomyocytes after H/R. Cardiomyocytes were treated with $\mathrm{H} / \mathrm{R}$ for different times. A. Western blot analysis was performed to determine the expression of nexilin. GAPDH was used as an internal control. B. Quantities for densitometric analysis of nexilin. Each bar represents the means \pm SD of 3 independent experiments. $* \mathrm{P}<0.05, * * \mathrm{P}<0.01$, compared with control.

\section{HGF upregulated nexilin expression in H/R-treated cardiomyocytes}

To determine the effect of HGF on nexilin expression in H/R-treated cardiomyocytes, Western blot and RT-PCR were performed. After 24-h treatment with HGF, Nexilin protein level significantly increased (Figure 2A). The nexilin mRNA level was also upregulated after HGF treatment in a dose-dependent manner (Figure 2B).

\section{SP600125 blocked HGF-induced nexilin upregulation in cardiomyocytes}

To clarify the mechanism involved in HGF-induced nexilin upregulation, SP600125, SB203580, PD 98059, and U0126 were used to treat the cells, along with HGF. After HGF treatment for $24 \mathrm{~h}$, nexilin expression was obviously increased, as shown in Figure 3. HGFinduced nexilin upregulation was partly blocked by SP600125, a JNK inhibitor. However, co-treatment with SB203580, a p38 MAPK inhibitor; PD 98059, an MAPKK inhibitor; and U0126, an MEK1/2 inhibitor, showed little effect on HGF-induced nexilin upregulation, indicating that only JNK pathway is involved in HGF-induced nexilin upregulation. 


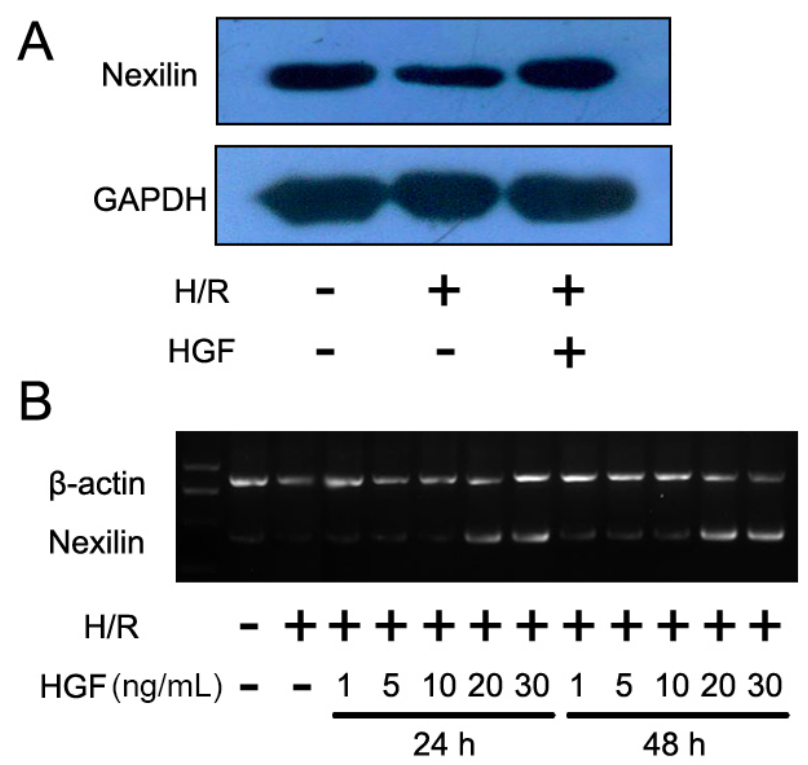

Figure 2. Nexilin expression was elevated by HGF in H/R-treated cardiomyocytes. Cardiomyocytes were cultured in a hypoxic incubator with or without indicated concentrations of HGF for $24 \mathrm{~h}$, and then cells were replaced to a normal condition for indicated times. A. Western blot analysis was performed to determine the expression of nexilin. B. RT-PCR was used to determine the mRNA levels of nexilin.

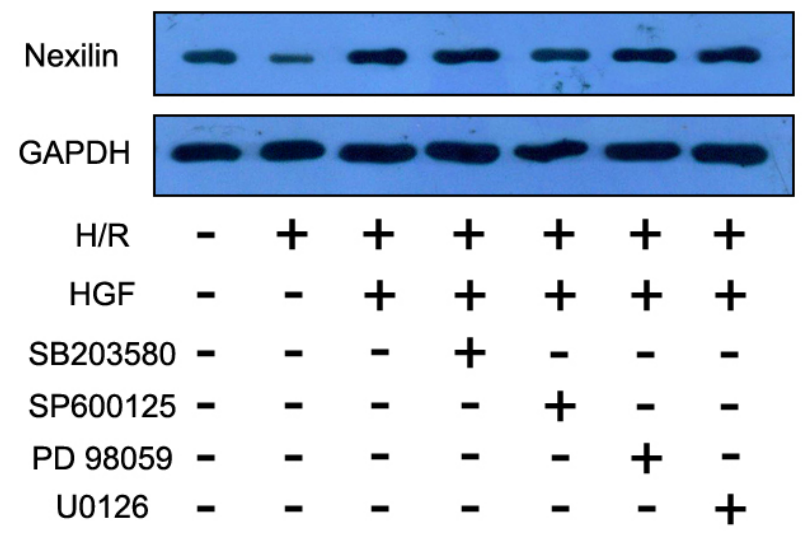

Figure 3. The upregulation of nexilin was mitigated by SP600125. Cardiomyocytes were treated with HGF (20 ng/ $\mathrm{mL})$ and SB203580 $(10 \mu \mathrm{M})$, SP600125 $(10 \mu \mathrm{M})$, PD98059 $(10 \mu \mathrm{M})$ or U0126 $(10 \mu \mathrm{M})$ in a hypoxic incubator for $24 \mathrm{~h}$, and then cells were replaced to a normal condition for another $24 \mathrm{~h}$. Western blot analysis was performed to determine the expression of nexilin.

\section{DISCUSSION}

In the present study, we demonstrated that HGF improved nexilin expression in H/R-treated cardiomyocytes. This effect was partly mitigated by a JNK inhibitor, SP600125, indicating that 
the protective effect of HGF on cardiomyocytes is mediated, at least partially, by the JNK pathway. Heart failure due to dilated cardiomyopathy is among the most common causes of cardiovascular mortality. Recently, Frank and Frey (2011) suggest that Z-discs are critical elements involved in dilated cardiomyopathy. Nexilin, a newly discovered Z-disc protein, plays an important role in the pathogenesis of cardiomyopathies. Expression of nexilin proteins encoded by NEXN mutant alleles or deficiency of nexilin in zebrafish results in Z-disk damage and heart failure, indicating a dominant-negative effect of nexilin mutations (Hassel et al., 2009; Frank and Frey, 2011). In the present study, we found that $\mathrm{H} / \mathrm{R}$ decreased the expression of nexilin mRNA and protein. The in vitro model of $\mathrm{H} / \mathrm{R}$ of cardiomyocytes mimics the in vivo ischemia-reperfusion injury of myocardia. Since there is no in vivo evidence about the expression of nexilin in the ischemia-reperfusion injury of myocardia yet, our data may provide a new insight into the understanding of myocardial ischemic injury. More interestingly, HGF treatment upregulated the expression of nexilin, indicating HGF may exert a protective role through modulating nexilin expression.

HGF has been shown as a cardioprotective agent due to its antiapoptotic effect on cardiomyocytes for many years (Nakamura et al., 2000). Accumulating evidence highlights a central role for MAPK family in the protective effect of HGF on cardiomyocytes (Nakamura et al., 2000; Kitta et al., 2001; Pietronave et al., 2010). However, these results are not consistent with each other. Nakamura et al. (2000) showed that HGF significantly increased phosphorylated ERK-1/2 in cardiomyocytes. Kitta et al. (2001) also found that HGF could activate MEK-ERK pathway and that pretreatment with PD98059 partially blocked HGF signaling for protection against hydrogen peroxide-induced cardiac myocyte apoptosis. However, a previous study (Wang et al., 2004) suggested that the inhibition of Bid/Bax-induced cell death by HGF primarily involved p38 MAPK, and in part, Akt-dependent pathways, but not ERK1/ ERK2 pathway. This inconsistency may be due to the difference in the cell types. In the present study, the inhibitor of JNK pathway, but not those of p38 MAPK, MAPKK, and MEK1/2 pathways, inhibited the expression of Nexilin, indicating that JNK pathway is involved in the HGF-mediated induction of nexilin expression.

In summary, our data suggest that HGF upregulates nexilin expression in H/R-treated cardiomyocytes, and that JNK pathway is involved, at least partially, in this process. To our knowledge, this is the first documentation of HGF upregulating nexilin expression through JNK pathway. However, this is an in vitro study. Further studies are needed to investigate whether HGF can exert similar effects in myocardial ischemic injury.

\section{Conflicts of interest}

The authors declare no conflict of interest.

\section{ACKNOWLEDGMENTS}

Research supported by the National Natural Science Foundation of China (NSFC, \#81260263).

\section{REFERENCES}

Chen XH, Minatoguchi S, Kosai K, Yuge K, et al. (2007). In vivo hepatocyte growth factor gene transfer reduces 
myocardial ischemia-reperfusion injury through its multiple actions. J. Card Fail. 13: 874-883.

Date T, Belanger AJ, Mochizuki S, Sullivan JA, et al. (2002). Adenovirus-mediated expression of p35 prevents hypoxia/ reoxygenation injury by reducing reactive oxygen species and caspase activity. Cardiovasc. Res. 55: 309-319.

Frank D and Frey N (2011). Cardiac Z-disc signaling network. J. Biol. Chem. 286: 9897-9904.

Hassel D, Dahme T, Erdmann J, Meder B, et al. (2009). Nexilin mutations destabilize cardiac Z-disks and lead to dilated cardiomyopathy. Nat. Med. 15: 1281-1288.

Kitta K, Day RM, Ikeda T and Suzuki YJ (2001). Hepatocyte growth factor protects cardiac myocytes against oxidative stress-induced apoptosis. Free Radic. Biol. Med. 31: 902-910.

Matsuda Y, Matsumoto K, Yamada A, Ichida T, et al. (1997). Preventive and therapeutic effects in rats of hepatocyte growth factor infusion on liver fibrosis/cirrhosis. Hepatology 26: 81-89.

Nakamura T, Nishizawa T, Hagiya M, Seki T, et al. (1989). Molecular cloning and expression of human hepatocyte growth factor. Nature 342: 440-443.

Nakamura T, Mizuno S, Matsumoto K, Sawa Y, et al. (2000). Myocardial protection from ischemia/reperfusion injury by endogenous and exogenous HGF. J. Clin. Invest. 106: 1511-1519.

Ohtsuka T, Nakanishi H, Ikeda W, Satoh A, et al. (1998). Nexilin: a novel actin filament-binding protein localized at cellmatrix adherens junction. J. Cell Biol. 143: 1227-1238.

Ozaki I, Zhao G, Mizuta T, Ogawa Y, et al. (2002). Hepatocyte growth factor induces collagenase (matrix metalloproteinase-1) via the transcription factor Ets-1 in human hepatic stellate cell line. J. Hepatol. 36: 169-178.

Pietronave S, Forte G, Locarno D, Merlin S, et al. (2010). Agonist monoclonal antibodies against HGF receptor protect cardiac muscle cells from apoptosis. Am. J. Physiol. Heart Circ. Physiol. 298: H1155-H1165.

Taniyama Y, Morishita R, Aoki M, Hiraoka K, et al. (2002). Angiogenesis and antifibrotic action by hepatocyte growth factor in cardiomyopathy. Hypertension 40: 47-53.

Wang H, Li Z, Wang J, Sun K, et al. (2010). Mutations in NEXN, a Z-disc gene, are associated with hypertrophic cardiomyopathy. Am. J. Hum. Genet. 87: 687-693.

Wang X, Zhou Y, Kim HP, Song R, et al. (2004). Hepatocyte growth factor protects against hypoxia/reoxygenationinduced apoptosis in endothelial cells. J. Biol. Chem. 279: 5237-5243.

Yaekashiwa M, Nakayama S, Ohnuma K, Sakai T, et al. (1997). Simultaneous or delayed administration of hepatocyte growth factor equally represses the fibrotic changes in murine lung injury induced by bleomycin. A morphologic study. Am. J. Respir. Crit. Care Med. 156: 1937-1944.: 1937-1944. 